Title:

Author(s):

Submitted to:
Prediction and Characterization of Complex Systems

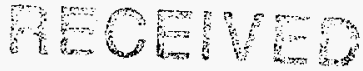

001111988

Ronnie Mainieri, T-13
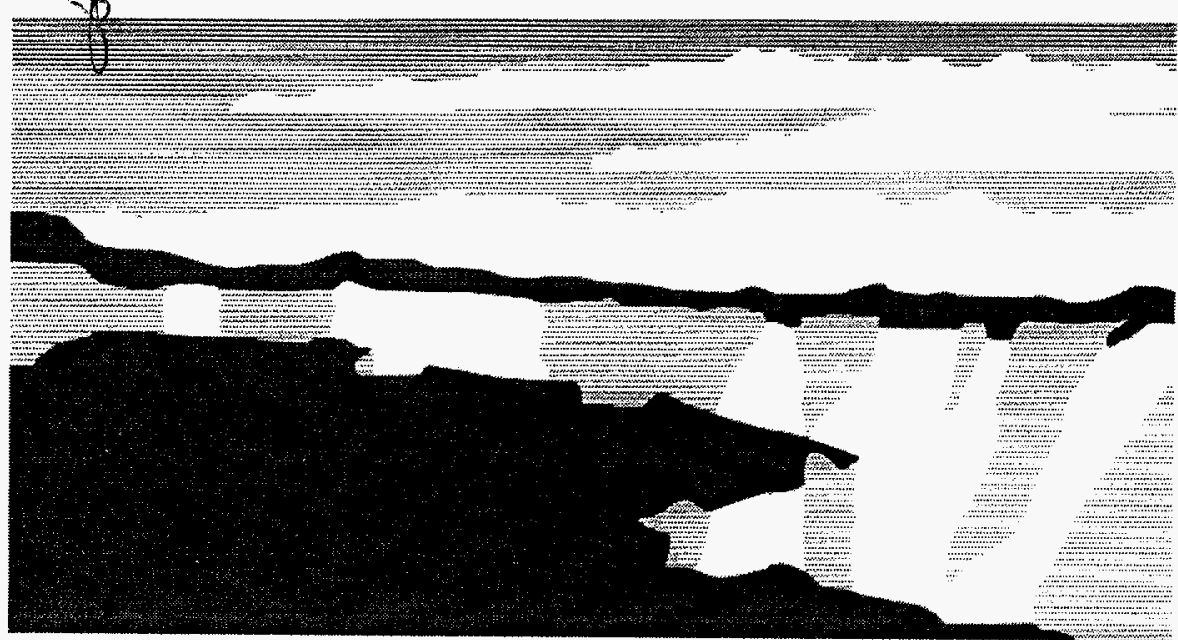

Los Alamos National Laboratory, an affirmative action/equal opportunity employer, is operated by the University of Califomia for the U.S. Department of Energy under contract W-7405-ENG-36. By acceptance of this article, the publisher recognizes that the U.S. Government retains a nonexclusive, royaltyfree license to publish or reproduce the published form of this contribution, or to allow others to do so, for U.S. Government purposes. The Los Alamos National Laboratory requests that the publisher identify this article as work performed under the auspices of the U.S. Department of Energy. 


\section{DISCLAIMER}

Portions of this document may be illegible in electronic image products. Images are produced from the best available original document. 


\title{
Prediction and Characterization of Complex Systems
}

Ronnie Mainieri*, Markus Baer (Fritz-Haber Institut), Helmut Brand (University of Bayreuth), John Budzinski (California Institute of Technology), Adi Buisara (Naval Command Control and Ocean Surveillance Center), Sergey Burtsev (Clarkson University), Gustavo Cruz-Pacheco (University of Arizona), Rajarshi Das (Santa Fe Institute), Robert Deissler (University of California Santa Cruz), Jan Finjord (Stavanger College), Christor Frousakis (I.C. Engines

\& Combustion Laboratory), Ivan Gjaja (University of Maryland), Niels Gottschalk (Fritz-Haber Institut), Veser Gotz (University of Minnesota), Aric Hagberg (University of Arizona), Gyorgy Haller (Brown University), Xiaowei He (Georgia Institute of Technology), Yun Huang (Peking University), Daniel Lathrop (Emory University), Yuanming Liu (University of Washington), John Lowenstein (New York University), Gottfried Mayer-Kress (Center for Complex Systems Research), Mark Millonas (University fo Texas at Austin), Christin Mira (Institut National des Sciences Appliques DGE), Melanie Mitchell (Indiana University), Werner Pesch (University of Bayreuth), Arkadi Pikovsky (Potsdam University), David Ropp (University of Arizona), Michael Schatz

(University of Texas at Austin), Mary Silber (California Institute of Technology), Ann Stanley (Iowa State University), Timothy Sullivan (Kenyon College), Nicholas Tufillaro (Bryn Mawr College), and Joseph Werne

(National Center for Atmospheric Research)

\begin{abstract}
This is the final report of a one-year, Laboratory-Directed Research and Development (LDRD) project at the Los Alamos National Laboratory (LANL). Complex systems are difficult to characterize and to simulate. By considering a series of explicit systems, through experiments and analysis, this project has shown that dynamical systems can be used to model complex systems. A complex dynamical system requires an exponential amount of computer work to simulate accurately. Direct methods are not practical and it is only by an hierarchical approach that one can gain control over the exponential behavior.
\end{abstract}

* Principal investigator, e-mail: ronnie@lanl.gov 
This allows the development of efficient methods to study fluid flow and to simulate biological systems. There are two steps in the hierarchical approach. First, one must characterize the complex system as a collection of large domains or objects that have their own forms of interactions. This is done by considering coherent structures, such as solitons, spirals, and propagating fronts and determining their interactions. Second, one must be able to predict the properties of the resulting low-dimensional dynamical system. This is accomplished by an understanding of the topology of the orbits of the dynamical system. The coherent structure description was carried out in fluid and reaction diffusion systems. It was shown that very simple models from statistical mechanics could characterize a rotating Rayleigh-Bénard system and that patterns in reaction-diffusion systems are well described by soliton-like solutions. The studies of dynamical systems showed that simple characterizations of the phase space can be used to determine long time bounds. Also, that periodic orbit theory can be used to demonstrate that Monte Carlo simulations will converge to incorrect results.

\section{Background and Research Objectives}

The study of complex systems can be understood mathematically as the study of dynamical systems: their evolution and their attractors. These dynamical systems may have an infinite number of dimensions and in some cases their equations may not be known explicitly. When the dynamics is chaotic, one cannot study them by following their evolution from a known initial condition. Due to sensitive dependence on initial conditions, small errors get amplified and prediction becomes unreliable.

Instead, chaotic systems are studied statistically. A special quantity, thought to be important in the dynamics of the system, is chosen-an observable-and its statistical properties computed from theory and determined from experiment; then they are compared. Because of the fundamental use of statistical quantities in the study of complex systems, probability distributions play a central role. This point of view was developed throughout the 20th century. Hadamard, Artin, Krylov, Kolmogorov, Sinai, Bowen, Ruelle, and Feigenbaum are a few of the exponents of this point of view for dynamical systems.

Many of the areas of complex systems can be understood as the study of dynamical systems and their invariant probability distributions. Time-series analysis is interested in determining the dynamical system that generated a signal. To make predictions from the timeseries, the invariant measure of that dynamical system must be determined. Studies of patterns 
formation in fluids are interested in describing the observed patterns in a simple manner (as a low-dimensional dynamical system) and how the observed features correlate to the behavior of parameters.

The probability distributions that arise in these and other complex systems have to be characterized and the processes by which they are generated described. In principle these probability distributions are Schwartzian distributions and they could be expanded in series of simpler distributions. But this has not proven to be an insightful approach. The probabilities are too complex and the series obtained converge too slowly-a better approach is needed.

We have proposed the study of complex systems through the chaotic dynamics that generate their characterizing probability distributions. We studied these problems in an interdisciplinary way, as the techniques and problems of one field often shed new light in the techniques and problems of another. The focus of this project in pattern formation and complex systems is on the understanding of complex dynamical systems: time-series analysis, pattern formation, and dynamical systems with and without noise.

\section{Importance to LANL's Science and Technology Base and National $R$ \& $D$ Needs}

Research in complex dynamical systems has great development potential. Physical problems, just like problems in computer science, can be divided into two large groups: those that require a polynomial time to solve, and those that require an exponential time. Complex dynamical systems research deals with the problems that require an exponential amount of work to solve on a computer. Just as there are no general algorithms to deal with nonpolynomial problems in computer science, we do not expect general methods for dealing with complex dynamical systems. What we do expect are efficient algorithms that solve the problem for all practical purposes. This analogy also explains the interdisciplinary nature of the research. Just as in computer science, one gains insight by posing the problem in different forms and areas.

The exponential character of the problems in complex dynamical systems implies that they are close to impossible to solve with a computer if naive methods are used. If we gain control over this exponential behavior, it may then be possible to design complex molecules in a computer, to develop efficient methods to study fluid flow, and to simulate biological systems.

Many of the Laboratory projects require large-scale simulations of complicated systems. Because of their size, it is not always possible to simulate these systems from first principles and time-saving techniques become essential. The methods developed and the techniques used 
have been made known to various divisions of the Laboratory through direct interactions with other investigators and through a series of seminars.

\section{Scientific Approach and Results to Date}

The approach used to accomplished the proposed work was varied. Analytical, computational, and experimental methods were used. The results are discussed grouped in two major categories: dynamical systems and pattern-forming systems. The project also supported a summer workshop in complex systems at the Santa Fe Institute. Interaction with participants of this workshop has proven fruitful. Also, many postdoctoral fellows that come to work at the Laboratory have been summer workshop alumni and alumnae.

\subsection{Dynamical Systems}

Many of the systems studied in physics are chaotic systems in disguise. The chaos manifests itself in the slow convergence of physical quantities. Disordered systems and localization, classical spin systems, transport in mesoscopic systems, and the electronic configuration of molecules are all examples of chaotic systems. The common thread among these systems is their description in terms of a transfer operator (transfer matrix, PerronFrobenius operator, trace of a Green's function). Because these systems are chaotic, they are dense with periodic orbits and the invariant measure of the operator can be understood in terms of these orbits. This leads to a cycle expansion and a rapidly converging series.

Two results in this area have been described $[1,15]$. By using the periodic orbits that exist within dynamical systems those authors where able to characterize them with a high degree of accuracy. In one publication [15], a large set of periodic orbits was extracted from an experiment and then used to pinpoint the theoretical model describing it. In the other publication [1], it was shown that Monte Carlo simulations can be very misleading when applied to dynamical systems. In a simple model that could be described by its periodic orbits, the Monte Carlo method was used to compute the same quantity being computed with the periodic orbits. Even though the Monte Carlo method had converged to an answer by all practical criteria, it converged to the wrong answer. If the more accurate periodic orbit calculation had not been available, one would have never been able to detect this subtle failure of the Monte Carlo method. This observation has led us to re-examine other Monte Carlo procedures.

Two other results in the area of dynamical systems involve the characterization of the resulting attractors. A method was developed that allows the efficient computation of Lyapunov exponents [12]. The method eliminates a computationally expensive diagonalization 
of the Jacobian matrix. With this method it now becomes feasible to compute the Lyapunov exponent for any system that can be solved numerically. It was also shown that the Lorenz attractor can be contained in a set described by simple geometrical figures [9]. This allows one to get a good and rigorous upper bound for the Lyapunov exponent of the Lorenz system. This is important as a check for the many algorithms being developed to estimate Lyapunov exponents.

\subsection{Pattern Formation}

Fluid dynamics is a high-dimensional dynamical system. To study the patterns formed in fluid motion one should, in principle, understand the high-dimensional fluid flow. Because the system is chaotic (turbulent in most cases), this is not practical. This is also counter-intuitive, because from observing the fluid flow experiments one can see that certain simple features dominate the dynamics. The problem in pattern formation has been in finding a precise description of these simple features. Phrased differently: Is there an effective low-dimensional dynamical system that captures most of the dynamics of the fluid flow and its patterns?

The study of patterns formation is a concrete problem that offers a test bed for the problems of characterizing high-dimensional dynamical systems and the measures they generate. It is also an open problem in physics. Patterns affect the transport properties in many physical systems and they must be accounted for. Unless there is a simple (lowdimensional) dynamics, this is not possible. Examples where patterns affect the physical properties of a system are: weather, diffusion, and conductivity properties in fluids.

In a breakthrough, a turbulent fluid system with coherent structures was described by a simple statistical model [10]. The model approximates the motion of the Earth's atmosphere and the formation of tornadoes. The number and size of these tornadoes were accurately described by a simple Ising model, where groups of spins in the same direction represent the tornadoes. This is the first time that a simple model has been constructed to describe a complex fluid system.

Simple models, like the one used by Ecke, et al., [10] cannot be well simulated on a computer. The reason is that one needs to use a very large grid or a large number of Fourier components. A rigorous estimate on how many modes must be used was discussed by Doering and Titi [8], where an upper bound for the power spectrum for the Navier-Stokes equation was derived.

In a series of articles $[6,7,2,3]$, it was established that soliton-like solutions exist even for two-dimensional dissipative systems. It was explained how they are formed and how these solitons interact. This research sheds light on the poor performance of platinum catalytic converters used in cars. A different type of pattern formation system has been studied [11, 
13]. An Ising-Bloch transition was shown to be the mechanism for the formation of spiral waves and propagating fronts in a reaction diffusion system. These systems occur in biological systems and in industrial chemical processes.

\section{Publications/References}

1. Bauer, Oliver and Ronnie Mainieri "The Convergence of Chaotic Integrals," Chaos, (accepted), Los Alamos Report LA-UR 95-0683 (1995).

2. Brand, Helmut and Robert Deissler, "The Interaction of Localized Solutions in a Surface Reaction," Conference on NEEDS 94, Center for Nonlinear Studies, Los Alamos National Laboratory, Los Alamos, NM, September 12-16, 1994, Los Alamos Report LA-UR 94-3529 (1994).

3. Brand, Helmut and Robert Deissler, "Stable Spatially Localized Solutions and Holes in Optical Bistability," Physica A, (submitted), Los Alamos Report LA-UR 95-1461 (1995).

4. Brandow, Baird, "A Valence-Fluctuation Mechanism with Highly Anisotropic S-Wave Pairing," in Proceedings of the Conference on Spectroscopies in Novel Superconductors, Stanford, CA, March 15-18, 1995, Journal of Physics and Chemistry of Solids, (submitted), Los Alamos Report LA-UR 95-0972 (1995).

5. Brown, Reggie, Nikolai Rulkov, and Nicholas Tufillaro, "Synchronization of Chaotic Systems: The Effects of Additive Noise and Drift in the Dynamics of the Driving," Physical Review E, (submitted), Los Alamos Report LA-UR 94-2181 (1994).

6. Deissler, Robert and Helmut Brand, "Interaction of Breathing Localized Solutions for Subcritical Bifurcations," Physical Review, (submitted), Los Alamos Report LA-UR 943524 (1994).

7. Deissler, Robert and Helmut Brand, "Two-Dimensional Localized Solutions for Subcritical Bifurcations in Systems with Broken Rotational Symmetry," Physical Review $E$, (submitted), Los Alamos Report LA-UR 94-3525 (1994).

8. Doering, Charles and Edriss Titi, "Exponential Decay Rate of the Power Spectrum for Solutions of the Navier-Stokes Equations," Physics of Fluids, (submitted), Los Alamos Report LA-UR 94-3710 (1994).

9. Doering, Charles and J. Gibbon, "On the Shape and Dimension of the Lorenz Attractor," Dynamics and Stability of Systems, (submitted), Los Alamos Report LA-UR 95-0900 (1995).

10. Ecke, Robert, Yuchou Hu, Ronnie Mainieri, and Guenter Ahlers, "Excitation of Spirals and Chiral Symmetry Breaking in Rayleigh-Benard Convection," Science, (submitted), Los Alamos Report LA-UR 95-0505 (1995).

11. Elphick, Christian, Aric Hagberg, and Ehud Meron, "Dynamic Front Transitions and Spiral-Vortex Nucleation," Physical Review E, (submitted), Los Alamos Report LA-UR 95-0017 (1995). 
12. Gjaja, Ivan, "Closed-Form Expressions for the Noncompact Part of SP(2n)," Comments in Physical Review Letters, (submitted), Los Alamos Report LA-UR 94-4080 (1994).

13. Hagberg, Aric, "Front Transition and Spiral-Vortex Nucleation," in Colorado Days, Boulder, CO March 17-18, 1995, Los Alamos Report LA-UR 95-0976 (1995).

14. Millonas, Mark and Chris Ray, "A Simple Homogeneours Model which Exhibits Glassy Relaxation," Physical Review Letters, (submitted), Los Alamos Report LA-UR 94-4054 (1994).

15. Molteno, T. and Nicholas Tufillaro, "An Experimental Investigation into the Dynamics of a String," Journal of Sound \& Vibration, (submitted), Los Alamos Report LA-UR 944053 (1994).

16. Shevitz, Daniel, Roger Jones, and Brad Paden, "Computational Issues in a Nonlinear Observer for Systems with Quantized Outputs," ASME Journal of Dynamics Systems, Measurement, and Control, (submitted), Los Alamos Report LA-UR 95-1631 (1995).

\section{DISCLAIMER}

This report was prepared as an account of work sponsored by an agency of the United States Government. Neither the United States Government nor any agency thereof, nor any of their employees, makes any warranty, express or implied, or assumes any legal liability or responsibility for the accuracy, completeness, or usefulness of any information, apparatus, product, or process disclosed, or represents that its use would not infringe privately owned rights. Reference herein to any specific commercial product, process, or service by trade name, trademark, manufacturer, or otherwise does not necessarily constitute or imply its endorsement, recommendation, or favoring by the United States Government or any agency thereof. The views and opinions of authors expressed herein do not necessarily state or reflect those of the United States Government or any agency thereof. 